\title{
ON FUNCTIONS WHICH ARE FOURIER TRANSFORMS
}

\section{R. E. EDWARDS}

1. Let $G$ be a locally compact and abelian group, $\bar{G}^{1}$ the dual group. Through this paper, $\overline{\mathscr{x}}^{1}$ will denote the set of all bounded Radon measure $\mu$ on $\bar{G}$; this set will be considered as a Banach algebra when provided with the customary norm as the dual of the space $C_{0}(\bar{G})$ defined below and with the ring product defined as convolution. If $\mu \in \overline{\mathscr{T}}^{1}$, its Fourier transform is by definition the bounded and continuous function on $G$ given by the formula

$$
T_{\mu}(x)=\int_{\bar{G}}(x, \bar{x}) d \mu(\bar{x}),
$$

where $(x, \bar{x})$ is the character function defining the duality between $G$ and $\bar{G} \cdot \overline{\mathcal{X}}^{1}$ contains as a closed ideal the set of functions on $\bar{G}$ which are summable for Haar measure, and an identification is possible provided one identifies a function and its equivalence class in $\bar{L}^{1}$ $=L^{1}(\bar{G})$. For $F$ in $\bar{L}^{1}, T_{F}(x)$ converges to zero at infinity on $G$. We shall denote by $T\left(\overline{\mathscr{X}}^{1}\right)$ the image of $\overline{\mathscr{X}}^{1}$ by the mapping $\mu \rightarrow T_{\mu} ; T\left(\bar{L}^{1}\right)$ is defined analogously.

We shall be concerned with several natural function spaces over a given locally compact space $K$, namely:

(a) the space $C_{0}(K)$ of all continuous functions on $K$ which tend to zero at infinity (a void condition if $K$ is compact); often we think of $C_{0}(K)$ as a Banach space equipped with the uniform norm.

(b) the space $K(K)$ of all continuous functions on $K$ having compact supports;

(c) the space $A(K)$ of all continuous, uniformly almost periodic functions on $K, K$ being assumed to be an abelian topological group.

Of these, (a) and (c) are both $C$-algebras in the sense defined in $\$ 2$ when equipped with the uniform norm, pointwise algebraic operations, and the involution defined as passage from a function $f$ to its complex conjugate $f^{*} ; C_{0}(K)$ has no unit unless $K$ is compact.

Segal [1] has shown that if $T\left(\bar{L}^{1}\right)$ exhausts $C_{0}(G)$, then $G$ is finite. More recently, Hewitt [2] has shown that the same conclusion follows from the hypothesis that $T\left(\overline{\mathscr{X}}^{1}\right)$ contains $A(G)$. The aim of this paper is to give a more direct proof of an assertion which contains the results of Segal and Hewitt as special cases, together with at least two other similar and interesting results.

Received by the editors March 23, 1953. 
I am indebted to Professor Hewitt for the oportunity of seeing his paper [2] in manuscript form. My thanks are due also to M. Grothendieck for several informal discussions and some observations which suggested the form of the main Theorem 1.

2. A general theorem. At the risk of enlarging the already rather complicated classification of Banach algebras, we shall find it convenient to term briefly a $C$-algebra any commutative, complete, normed algebra over the complex field, with or without a unit, which is self-adjoint in the usual sense and for which there is a constant $K>0$ such that

$$
\left\|f^{2}\right\| \geqq K\|f\|^{2}
$$

for all $f$ in the algebra. There are two well known criteria which ensure that a commutative, complete, normed complex algebra $R$ is a $C$-algebra, namely:

$\left(c_{1}\right) R$ has a unit and is equipped with an involution $f \rightarrow f^{*}$ with the customary properties and for which further

$$
\left\|f f^{*}\right\|=\|f\|^{2} \quad(f \in R) .
$$

$\left(c_{2}\right) R$ is equipped with an involution $f \rightarrow f^{*}$ such that $f f^{*}$ has a quasi-inverse for all $f \in R$; and (1) is valid.

The name $C$-algebra is perhaps justified by the first of the following two lemmas, both of which are proved in all essentials by Gelfand and Neumark [3, Lemmas 1 and 2].

Lemma 1. Let $R$ be a $C$-algebra. Then $R$ is algebraically and topologically isomorphic with the C-algebra $C_{0}(K)$, where $K$ is the locally compact space of characters of $R$.

Lemma 2. Let $R$ be as in Lemma 1. Let $R^{\prime}$ be a normed complex algebra, not necessarily complete. Suppose further that in $R^{\prime}$ there are no generalised nilpotents other than zero. If $R$ and $R^{\prime}$ are algebraically isomorphic, then $R^{\prime}$ is complete and the said isomorphism is also topological.

We are now ready to state and prove our main theorem.

Theorem 1. Let $R$ be a $C$-algebra, $\bar{G}$ a locally compact and abelian group. Suppose there exists an algebraic isomorphism $u$ of $R$ onto a subalgebra $R$ of $\overline{\mathcal{X}}^{1}$. Then $R$ is of finite dimension.

PROOF. Since there are no generalised nilpotents in $\overline{\mathcal{X}}^{1}$ other than zero, the same is true a fortiori of $R$. From Lemma 2 we conclude 
that $u$ is also a topological isomorphism; in particular, $R$ and $R$ are isomorphic as Banach spaces.

Now suppose if possible that $R$ were of infinite dimension. Take any infinite sequence $\left\{f_{n}\right\}_{n=1}^{\infty}$ of linearly independent elements of $R$. Choose for each $n$ an element $f_{n}^{*}$ of $R$ symmetric to $f_{n}$, and let $R_{0}$ be the closed subalgebra of $R$ generated by the $f_{n}$ and the $f_{n}^{*}$. It is then clear that $R_{0}$ is a separable $C$-algebra. $u$ sets up a Banach space isomorphism between $R_{0}$ and its image $R_{0}=u\left(R_{0}\right)$ in $\overline{\mathscr{X}}^{1}$.

It follows that $R_{0}$ is a separable subspace of $\overline{\mathcal{X}}^{1}$ and so, as a consequence of the Lebesgue-Radon-Nikodym theorem, is isomorphic as a Banach space with a closed vector subspace of the $L^{1}$-space constructed relative to some positive Radon measure. Hence, by another application of the Lebesgue-Radon-Nikodym theorem, $R_{0}$ is sequentially weakly complete. The same is therefore true of its isomorph $R_{0}$.

On the other hand, Lemma 1 tells us that $R_{0}$ is isomorphic with the space $C_{0}(K)$, where $K$ is the locally compact and metrisable space of characters of the separable algebra $R_{0}$. And, as was pointed out to me by $\mathrm{M}$. Grothendieck, such a space $C_{0}(K)$ is sequentially weakly complete if and only if $K$ is finite. In fact, the condition implies that $K$ is discrete, hence finite if it is compact. If $K$ is not compact, we argue by observing that, since $C_{0}(K)$ is separable, $K$ satisfies the second axiom of countability; hence $K$ is $\sigma$-compact. Take then a countable basis $\left\{E_{n}\right\}_{n=1}^{\infty}$ for the compact subsets of $K$; for each $n$ there is then a function $\phi_{n}$ in $C_{0}(K)$ taking its values in $[0,1]$ and equal to 1 on $E_{n}$. The sequence $\left\{\phi_{n}\right\}_{n=1}^{\infty}$ is then a weak Cauchy sequence in $C_{0}(K)$, but its pointwise limit is the constant function 1 on $K$; the sequence $\left\{\phi_{n}\right\}_{n=1}^{\infty}$ is therefore not weakly convergent. This contradiction establishes the assertion.

Finally, since $K$ is finite, $R_{0} \simeq C_{0}(K)$ is of finite dimension, contrary to its construction. This completes the proof.

REMARK. If one assumes either that $u^{-1}$ is continuous, or that $R$ is closed in $\overline{\mathscr{x}}^{1}$ and that $u$ is continuous, one could use the Banach inversion theorem and so dispense entirely with the assumption that $R$ is a $C$-algebra. It would suffice that $R$ be a Banach space and that $u$ be linear. The conclusion would of course be correspondingly weakened to $R$ being sequentially weakly complete. This type of argument would in fact suffice for the applications we have in mind, but it seems neater to have the stronger conclusion once for all.

3. Applications. To obtain an extension of Segal's theorem, one takes an arbitrary subset $A$ of $G$ and considers the algebra $R$ 
$=C_{0}(G, A)$ of all functions $f \in C_{0}(G)$ which vanish on $C A . R$ is equipped with the pointwise algebra, the uniform norm, and the involution defined as passage from $f$ to its complex conjugate $f^{*}$. Plainly $R$ is a $C$-algebra, the conditions $\left(\mathrm{c}_{2}\right)$ being satisfied. If then $C_{0}(G, A)$ is a subset of $T\left(\overrightarrow{\mathscr{X}}^{1}\right)$, we conclude from Theorem 1 that the interior of $A$ is finite. Thus we may state

THEOREM 2. If $G$ and $\bar{G}$ are as in $\$ 1$, and if every continuous function on $G$ which tends to zero at infinity and which vanishes outside a prescribed subset $A$ of $G$ belongs to $T\left(\overline{\partial \mathcal{T}}^{1}\right)$, then the interior of $A$ is finite. Segal's theorem obtains on taking $A=G$.

We pass next to the consideration of an extension of Hewitt's theorem concerning uniformly almost periodic functions. For this take a subgroup $\bar{H}$ of $\bar{G}$, and take for $R$ the subalgebra of $A(G)$ formed of those functions in $A(G)$ whose spectra are contained in $\bar{H}$. If every such function were the transform of a bounded measure on $\bar{G}$, Theorem 1 would show that the set of such functions is of finite dimension: this is true if and only if $\bar{H}$ is finite. Thus we have

THEOREM 3. $G$ and $\bar{G}$ being as in $\$ 1$, if every continuous, uniformly almost periodic function on $G$ having its spectrum contained in a preassigned subgroup $\bar{H}$ of $\bar{G}$ is the transform of a bounded Radon measure on $\bar{G}$, then $\bar{H}$ is finite.

A third, and I believe new, result of a similar character is

THEOREM 4. If $G$ and $\bar{G}$ are as in $\S 1$, and if there exists a neighborhood $U$ of the neutral element of $G$ with the property that every continuous function on $G$ vanishing outside $U$ is the transform of a bounded measure on $\bar{G}$, then $G$ is discrete.

Proof. The set $R$ of continuous functions on $G$ which are bounded and vanish outside $U$ is a $C$-algebra with the symmetry defined as passage to the complex conjugate function: this follows from criterion $\left(c_{2}\right)$. Our hypothesis and Theorem 1 together imply that the interior of $U$ is finite, and thus that $G$ is discrete.

4. Functions belonging locally to $T\left(\overline{\mathscr{X}}^{1}\right)$. We shall say that a given function $f$ on $G$ belongs locally to $T\left(\overline{\mathscr{X}}^{1}\right)$ if for each point $p$ of $G$ there is a neighbourhood $V$ of $p$ and a measure $\mu \in \overline{\mathcal{X}}^{1}$ such that $T_{\mu}(x)=f(x)$ for $x$ in $V$. Plainly, it is the same thing to assert that $f$ belongs locally to $T\left(\bar{L}^{1}\right)$ : this is so because $\bar{L}^{1}$ is an ideal in $\overline{\mathscr{X}}^{1}$, because the mapping $\mu \rightarrow T_{\mu}$ carries convolutions into pointwise products, and because there are functions $F$ in $\bar{L}^{1}$ such that $T_{F}(x)=1$ for $x$ in any preassigned compact subset of $G$. 
The problem of characterising completely and directly those functions on $G$ which belong locally to $T\left(\overline{\mathscr{H}}^{1}\right)$ seems to be one of extreme difficulty. However, from Theorem 4 one can deduce the following general negative result:

THEOREM 5. $G$ and $\bar{G}$ being as in $\$ 1$, if every continuous function on $G$ belongs locally to $T\left(\overline{\mathscr{X}}^{1}\right)$, then $G$ is discrete. The converse is true and trivial.

Proof. Let us show in fact that our hypothesis implies that every function $f$ in $K(G)$ belongs globally to $T\left(\bar{L}^{1}\right)$. Suppose $K$ is a compact subset of $G$ outside which $f$ is zero. By hypothesis, for each point $z$ of $K$ there is a relatively compact open neighbourhood $V(z)$ of $z$ and a function $F_{z}$ in $\bar{L}^{1}$ such that $f(x)=T_{F_{z}}(x)$ for $x$ in $V(z)$. Since $K$ is compact, we may choose a finite number of points $z_{i}(1 \leqq i \leqq n)$, such that the $V\left(z_{i}\right)$ already cover $K$. As is well known, there are then functions $H_{i}$ in $\bar{L}^{1}$ such that $T_{H_{i}}(x)=0$ for $x \in \mathcal{C} V\left(z_{i}\right), 1 \leqq i \leqq n$, and such that $\sum_{i=1}^{n} T_{H_{i}}(x)=1$ for $x$ in $K$. Then for all $x$ in $G$

$$
\begin{aligned}
f(x) & =f(x) \cdot \sum_{i=1}^{n} T_{H_{i}}(x)=\sum_{i=1}^{n} f(x) T_{H_{i}}(x) \\
& =\sum_{i=1}^{n} T_{F_{s_{i}}}(x) T_{H_{i}}(x)=T_{F}(x),
\end{aligned}
$$

where $F=\sum_{i=1}^{n}\left(F_{z_{i}} * H_{i}\right) \in \bar{L}^{1}$. Thus $f$ belongs globally to $T\left(\bar{L}^{1}\right)$. We have now only to apply Theorem 4 .

REMarks. (i) The proof shows incidentally that if $G$ is compact, a function on $G$ belongs locally to $T\left(\overline{\mathscr{X}}^{1}\right)$ if and only if it belongs globally to $T\left(\bar{L}^{1}\right)$ : in other words, $f$ belongs locally to $T\left(\overline{\mathscr{A}}^{1}\right)$ if and only if the Fourier transform

$$
\bar{f}(\bar{x})=\int_{G} f(x) \overline{(x, \bar{x})} d x
$$

of $f$ is summable over $\bar{G}$. This is of course just an extension of a classical result of Wiener concerning Fourier series (see, for example, $Z$ ygmund $[4$, p. 140]). Thus, for the case in which $G$ is the compact circle group, the classical theory of Fourier series exhibits many necessary conditions in order that a function shall belong locally to $T\left(\overline{\mathscr{X}}^{1}\right)$, together with a number of sufficient conditions. The most interesting such conditions are naturally ones which are not obviously generalisable to an arbitrary compact abelian group.

(ii) For the case in which $G$ is the real line, Dr. F. Smithies produced an independent proof that not all continuous functions belong 
locally to $T\left(\overline{\mathscr{X}}^{1}\right)$, based upon the remark that if a function $f(x)$ $(-\infty<x<\infty)$ coincides on a neighbourhood of $x=0$ with a function in $T\left(\overline{\mathscr{X}}^{1}\right)$, then, for any fixed $c>0$ one has

$$
f(0)=\lim _{a \rightarrow \infty} \int_{-c}^{c} f(x) \frac{\sin 2 \pi a x}{\pi x} d x .
$$

If this were the case for every continuous $f$, the Banach-Steinhaus theorem would entail that the numbers

$$
\int_{-c}^{c}|\sin 2 \pi n x / \pi x| d x \quad(n=1,2,3, \cdots)
$$

would be bounded with respect to $n$, which is plainly false. Actually, the formula (3) shows a little more. For, if we suppose that $f$ has period $2 \pi$, and if $\mathfrak{S}(f)$ denotes the complex Fourier series of $f$ and $\mathfrak{S}_{n}(f ; x)$ the $n$th symmetric partial sum of $\mathfrak{S}(f)$ at $x$, then one has the standard formula

$$
\Im_{n}(f ; 0)=(1 / 2 \pi) \int_{-\pi}^{\pi} f(x) \frac{\sin (n+1 / 2) x}{\sin (x / 2)} d x .
$$

By the Riemann-Lebesgue lemma applied to the difference of the integrals in the right members of (3) and (4), it follows that if (3) is true, then $\lim _{n \rightarrow \infty} \mathfrak{S}_{n}(f ; 0)=f(0)$, so that the Fourier series of $f$ must be convergent for $x=0$. However, given any positive continuous function $p(x)$ such that $p(x) / x$ is not summable over any neighbourhood of $x=0$, it is known [4, p. 173] that there exist continuous periodic functions $f$ such that

$$
|f(x)-f(0)| \leqq p(x)
$$

for small $|x|$, and yet which possess Fourier series which diverge at $x=0$. Any such function fails to be, locally at $x=0$, a member of $T\left(\overline{\mathscr{X}}^{1}\right)$.

(iii) In the reverse direction, it is clearly an advance to have sufficient conditions on a function $f$, supposed to belong to $L^{p}(G)$ for some $1 \leqq p \leqq 2$, in order that its transform $\bar{f}$ be summable over $\bar{G}$. For the case in which $G$ is the real line, numerous such sufficient conditions are known: many are analogues of results on Fourier series, and a number are to be found in [5], especially pp. 529-543. We take the trouble to record the following result, which is weaker than results obtained by Hille and Tamarkin but which gives also a useful inequality. The proof, which consists in essentials of an integration by parts followed by use of the Hölder inequality, will be omitted. 
LEMma 3. Let $f$ be a function on the real line such that there exist two numbers $p$ and $q, 1<p, q \leqq 2$, for which:

(i) $f \in L^{p}(-\infty, \infty)$;

(ii) $f$ is absolutely continuous over every bounded interval, and the derivative $f^{\prime} \in L^{q}(-\infty, \infty)$.

Then the Fourier transform $F$ of $f$ is summable. Further, there exists a number $M$, depending only on $p$ and $q$, such that

$$
\|F\|_{1} \leqq M \cdot\|f\|_{p}^{p q /(p q-p+q)} \cdot\left\|f^{\prime}\right\|_{q}^{q /(p q-p+q)} .
$$

This lemma is an extension of an inequality of Carlson-Beurling $[6 ; 7]$ which deals with the case $p=q=2$.

5. Concluding remarks. It is clear that the arguments used in proving Theorem 1 are not particularly related to the fact that we have under discussion a group and the Fourier transformation. An analogous argument might take place each time that $\bar{G}$ is replaced by a locally compact semigroup $M$, for example; $G$ would then be replaced by a suitable set $M^{*}$ of bounded continuous characters of $M . \mathcal{X}^{1}(M)$ is formed into an algebra when the convolution of two measures $\mu$ and $\nu$ is defined as a measure by the formula

$$
\begin{aligned}
\int_{M} \phi d(\mu * \nu) & =\int_{M \times M} \phi(x y) d(\mu \otimes \nu)(x, y) \\
& =\int_{M} d \mu(x) \int_{M} \phi(x y) d \nu(y) \\
& =\int_{M} d \nu(y) \int_{M} \phi(x y) d \mu(x)
\end{aligned}
$$

for $\phi$ a general member of $K(M)$. The essential point is that $M^{*}$ shall contain enough characters $\chi$ of $M$ in order that $\mu \in \mathcal{X}^{1}(M)$ and $\int_{M} \chi(x) d \mu(x)=0$ for all $\chi$ in $M^{*}$ shall imply that $\mu=0$. The Fourier transformation is then replaced by the mapping $T: \mu \rightarrow T_{\mu}(\chi)$ $=\int_{M} \chi(x) d \mu(x)$, carrying measures into functions on $M^{*}$. The details of such modifications may be left to the reader. To quote but a single example, one might take for $M$ the compact interval $[0,1]$, forming a semigroup under multiplication, and for $M^{*}$ the characters of the form $x \rightarrow x^{n}(n=0,1,2, \cdots)$. In this case the analogue of Theorem 1 would assert that any $C$-algebra (under pointwise operations) of Stieltjes moment sequences is necessarily of finite dimension. 


\section{REFERENCES}

1. I. E. Segal, The class of functions which are absolutely convergent Fourier transforms, Acta Sci. Math. Szeged. vol. 12 (1950) pp. 157-161.

2. E. Hewitt, Representation of functions as absolutely convergent Fourier-Stieltjes transforms, to appear.

3. I. Gelfand and M. Neumark, On the imbedding of normed rings into the ring of operators in Hilbert space, Rec. Math. N.S. vol. 12 (1943) pp. 197-213.

4. A. Zygmund, Trigonometrical series, Warsaw-Lw6w, Monografje Matematyczne, 1935.

5. E. Hille and J. D. Tamarkin, On the summability of Fourier series. III, Math. Ann. vol. 108 (1933) pp. 525-577.

6. F. Carlson, Une inégalité, Arkiv för Matematik, Astronomi och Fysik vol. 25B (1934) pp. 1-5.

7. A. Beurling, Sur les integrales de Fourier absolument convergentes et leur application d une transformation fonctionelle, Proc. 9th Scand. Math. Congress 1938, Helsingfors, 1939, pp. 345-366.

Nancy, France 